International Conference on Ceramics, Bikaner, India

International Journal of Modern Physics: Conference Series

Vol. 22 (2013) 736-740

(C) World Scientific Publishing Company

DOI: $10.1142 / \mathrm{S} 2010194513010957$

\title{
LIQUID CRYSTALS AND APPLICATIONS OF CHLOSTERIC LIQUID CRYSTAL IN LASER
}

\author{
SURESH SONI, DIMPLE DHARNIYA BISHNOI, SUBHASH SONI, RAMSWROOP \\ Deptt. of Physics, Khalsa Girls College, Sadulshahar-335062, (raj.), India \\ Deptt. of Physics, Seth G.L.Bihani S.D.P.G.College, Sriganganagar-335001, (raj.), India \\ Lecturer in Physics, Govt.Sr.Sec.School, Rawatsar-335524, (raj.), India \\ Project Coordinator, North Bengal Science Centre, Siliguri-734010, (West Bengal), India \\ Email: ddharnia34@gmail.com
}

\begin{abstract}
Liquid crystals are a state of matter intermediate between that of a crystalline solid and an isotropic liquid. They possess many of the mechanical properties of a liquid, e.g. - high fluidity, inability to support shear, formation, and coalescence of droplets. At the same time they are similar to crystals in that they exhibit anisotropy in their optical, electrical, and magnetic properties. We discuss some physical properties of nematic, cholesteric, and smectic liquid crystals (Specially focused on cholesteric) and some applications in laser due to Cholesteric Liquid Crystal's resonant cavity which formed spontaneously and intrinsically, in the form of selfassembled chiral nematic helix.
\end{abstract}

\section{Introduction}

Liquid crystals have become ubiquitous in everyday applications ranging from miniature mobile telephones to high-definition flat-panel displays. Such technologies are based on the reorientation of a liquid-crystal material in response to applied electric fields, which results in a change in its observed optical properties. The majority of present commercial displays are based on liquid crystals exhibiting a nematic phase - the least ordered mesophase. However, materials similar to the cholesterol derivatives studied by Austrian botanist Frederick Reinitzer.

Cholesteric liquid-crystal laser can be used as an optic fiber-based temperature sensor[4]. Cholesteric Liquid Crystals can be used in Flexible mirror less Laser[2]. Ferroelectric Liquid Crystal can be used in Electrically tunable waveguide Laser.

\section{Basics}

\section{Liquid Crystal}

Liquid crystals are wonderful materials. In addition to the solid crystalline and liquid phases, liquid crystals exhibit intermediate phases where they flow like liquids, yet possess some physical properties characteristic of crystals. Materials that exhibit such unusual phases are often called mesogens (i.e., they are mesogenic), and the various phases in which they could exist are termed mesophases. The well-known and widely 
studied ones are thermotropics, polymerics, and lyotropics. As a function of temperature, or depending on the constituents, concentration, substituents, and so on, these liquid crystals exist in many so-called mesophases- nematic, cholesteric, smectic, and ferroelectric.

A classification of liquid crystals based on their structural properties was first proposed by G. Friedel (1922), and they are generally divided into three main classes:

\subsection{Nematic}

A simplified picture of the relative arrangement of the molecules in the nematic phase is shown in Fig. (1.1). The locally preferred direction may vary throughout the medium, although in the unstrained (equilibrium) nematic, it does not. Much of the interesting phenomenology of liquid crystals involves the geometry and dynamics of the preferred axis, and so it is useful to define a vector field $n(r)$ giving its local orientation. This vector is called the director. Since its magnitude has no significance, it is taken to be unity. The molecules appear to be able to rotate about their long axes and also there seems to be no preferential arrangement of the two ends of the molecules.

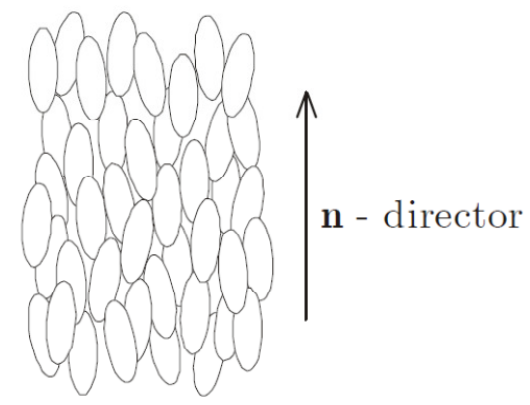

Fig 1.1. The nematic phase.

\subsection{Cholesteric Liquid Crystal}

The cholesteric phase is like the nematic phase in having long-range orientational order and no long-range order in the positions of the centers of mass of molecules. It differs from the nematic phase in that the director varies in direction throughout the medium in a regular way. The periodicity length of the cholesteric is actually only half this distance since $n$ and $-n$ are indistinguishable. For some reason, the structure of cholesterics is frequently referred to as a helix (with the appropriate pitch and axis); this usage has metaphoric value but little physical content.

A nematic liquid crystal is just a cholesteric of infinite pitch, and is not really an independent case. In particular, there is no phase transition between nematic and cholesteric phases in a given material, and nematic liquid crystals doped with enantiomorphic (not mirror symmetric) materials become cholesterics of long (but finite) pitch. 
The pitch of the common cholesterics is of the order of several thousand angstroms, and thus comparable with the wavelength of visible light. The spiral arrangement is responsible for the characteristic colors of cholesterics in reflection (through Bragg refiection by the periodic structure) and their very large optical rotatory power. The pitch can be quite sensitive to temperature, flow, chemical composition, and applied magnetic or electric fields.

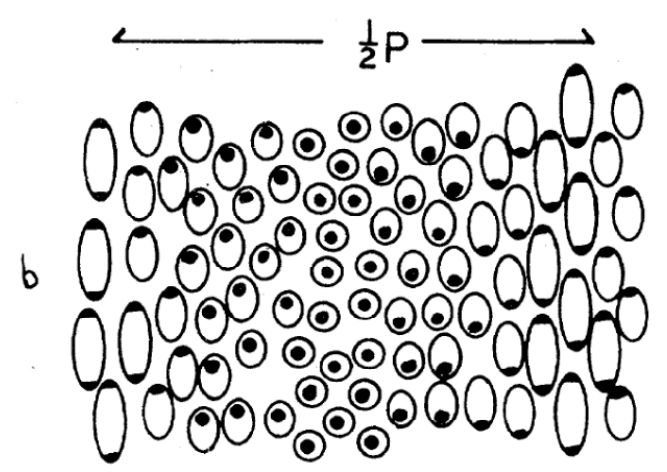

Fig 1.2. The cholesteric phase. The molecules tend to have the same alignment which varies regularly through the medium with a periodicity distance $\mathrm{p} / 2$. The positions of the molecules are not correlated.

\section{Use of cholesteric liquid crystal in Flexible mirrorless laser}

A cholesteric liquid crystal is a type of liquid crystal with a helical structure and which is therefore chiral. Cholesteric liquid crystals are also known as chiral nematic liquid crystals. They organize in layers with no positional ordering within layers, but a director axis which varies with layers. The variation of the director axis tends to be periodic in nature. The period of this variation (the distance over which a full rotation of $360^{\circ}$ is completed) is known as the pitch, p. The pitch varies with temperature and it can also be affected by the boundary conditions when the chiral nematic liquid crystal is sandwiched between two substrate planes.

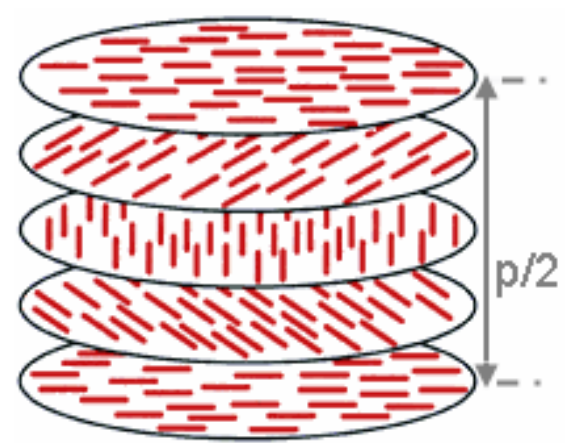

Fig 1.3. Half pitch of Cholesteric liquid Crystal 
Cholesteric liquid crystal (CLC) and chiral smectic liquid crystals such as ferroelectric liquid crystal, which have aperiodic helical structure with a periodicity of the optical wavelength, can be regarded as a one-dimensional Photonic Crystal. If liquid crystal has a chirality in the molecular structure, they form a 1D periodic helical structure spontaneously. In the CLC, liquid crystal molecules align their molecular long axes (director) homogeneously in the plane perpendicular to the helical axis and rotate the direction of director continuously along the helical axis. In such liquid crystals with the helical structure, a circularly polarized light with the same handedness as the helix propagating along a helical axis is selectively reflected (selective reflection) and a stop band appears. The wavelength $\lambda$ of the stop band is by,

$$
\lambda=n p
$$

where $n$ and $p$ are an averaged refractive index of liquid crystal and the pitch of helix, respectively,

$$
n=\frac{\sqrt{n o^{2}+n e^{2}}}{2}
$$

where no and ne are ordinary and extraordinary refractive indices of liquid crystal, respectively.

\section{Temperature dependence}

From the practical point of view, the thermal stability is important for the laser action. Figure 1.4 shows the temperature dependence of the emission peak wavelength of PCLC (closed symbols) and unpolymerized CLC mixture (open Symbols).

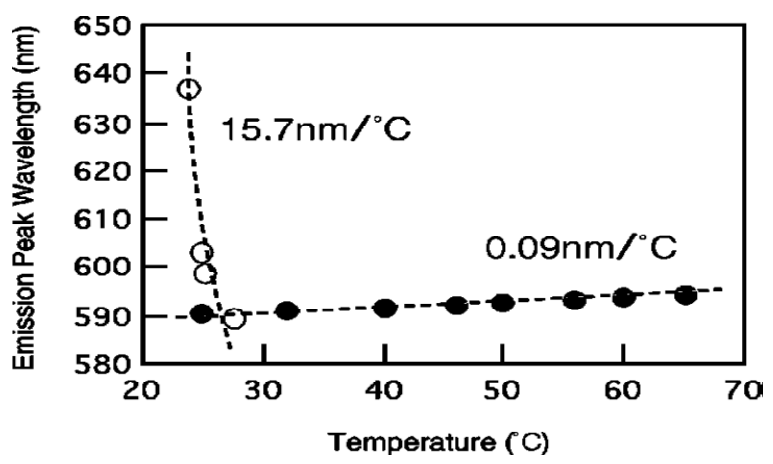

Fig 1.4. Temperature dependence of the lasing emission peak wavelength of the PCLC film (closed symbols) and unpolymerized CLC mixture (open Symbols).

Moreover, the helical pitch of the PCLC has no temperature dependence in contrast to that of unpolymerized cholesteric liquid crystal. This means that the operation wavelength of laser action is thermally stable, which is the great advantage for the device application. 


\section{References}

1. C. J. Newsome, M. O’Neill, R. J. Farley, and G. P. Bryan-Brown, Appl. Phys. Lett. 72, 2078 (1998)

2. Tatsunosuke Matsui, Ryotaro Ozaki, Kazuhiro Funamoto, Masanori Ozaki, and Katsumi Yoshino, Appl. Phys. Lett. 81, 3741 (2002)

3. Masahiro Kasano, Masanori Ozaki, Katsumi Yoshino, Dirk Ganzke, and Wolfgang, Appl. Phys. Lett. 82, 4026 (2003)

4. M. F. Moreira, I. C. S. Carvalho, W. Cao, C. Bailey, B. Taheri et al. , Appl. Phys. Lett. 85, 2691 (2004)

5. P.J.W. Hands, C.A. Dobson, S.M. Morris, M.M. Qasim, D.J. Gardiner, T.D. Wilkinson, H.J. Coles , Proc. SPIE 8114-28, Liquid Crystals XV, (2011)

6. Igor P. Ilchishin, Longin N. Lisetski, and Taras V. Mykytiuk, 1 December 2011 / Vol. 1, No. 8 / OPTICAL MATERIALS EXPRESS 1484

7. Damian J. Gardiner, Stephen M. Morris, Philip J. W. Hands, Carrie Mowatt, Rupert Rutledge, Timothy D. Wilkinson,1 and Harry J. Coles, January 2011 / Vol. 19, No. 3 / OPTICS EXPRESS 2432

8. Thresholdless Switching in Ferro- and Antiferro-electric Liquid Crystal Displays (Artur Adamski) (2004-2005) 